\title{
Membrane-assisted viral DNA ejection
}

Isaac Santos-Pérez ${ }^{\text {a }}$, Hanna M. Oksanen ${ }^{\text {b }}$, Dennis H. Bamford ${ }^{\text {b }}$, Felix M. Goñi ${ }^{\text {c }}$, David Reguera $^{\text {d }}$, Nicola G.A. Abrescia ${ }^{\text {a,e,* }}$

${ }^{a}$ Structural Biology Unit, CIC bioGUNE, CIBERehd, Bizkaia Technology Park, 48160 Derio, Spain.

${ }^{\mathrm{b}}$ Institute of Biotechnology and Department of Biosciences, Viikki Biocenter, University of Helsinki, P.O. Box 56, Viikinkaari 9B, 00014 University of Helsinki, Finland.

${ }^{\mathrm{c}}$ Unidad de Biofísica (CSIC, UPV/EHU) and Departamento de Bioquímica, Universidad del País Vasco, Bilbao, Spain.

${ }^{\text {d }}$ Departament de Física de la Materia Condensada, Facultat de Física, Universitat de Barcelona, 08028 Barcelona, Spain.

${ }^{\mathrm{e}}$ IKERBASQUE, Basque Foundation for Science, 48013 Bilbao, Spain.

*To whom correspondence should be addressed. Email: nabrescia@cicbiogune.es 


\begin{abstract}
Genome packaging and delivery are fundamental steps in the replication cycle of all viruses. Icosahedral viruses with linear double-stranded DNA (dsDNA) usually package their genome into a preformed, rigid procapsid using the power generated by a virus-encoded packaging ATPase. The pressure and stored energy due to this confinement of DNA at a high density is assumed to drive the initial stages of genome ejection. Membrane-containing icosahedral viruses, such as bacteriophage PRD1, present an additional architectural complexity by enclosing their genome within an internal membrane vesicle. Upon adsorption to a host cell, the PRD1 membrane remodels into a proteo-lipidic tube that provides a conduit for passage of the ejected linear dsDNA through the cell envelope. Based on volume analyses of PRD1 membrane vesicles captured by cryo-electron tomography and modeling of the elastic properties of the vesicle, we propose that the internal membrane makes a crucial and active contribution during infection by maintaining the driving force for DNA ejection and countering the internal turgor pressure of the host. These novel functions extend the role of the PRD1 viral membrane beyond tube formation or the mere physical confinement of the genome. The presence and assistance of an internal membrane might constitute a biological advantage that extends also to other viruses that package their linear dsDNA to high density within an internal vesicle.
\end{abstract}

\title{
Keywords
}

Models of viral DNA packaging; viral DNA delivery; lipid-containing viruses; membrane vesicle and properties; biophysical modeling; cryo-electron tomography. 


\section{Introduction}

In the course of evolution, viruses have developed different strategies for efficient delivery of their genome into the host cell. One of the best studied examples is that employed by the tailed dsDNA bacteriophages that typically package their genome at very high densities within a rigid proteinaceous capsid shell (Fig. 1A). The elastic, electrostatic, entropic, osmotic, and hydration energetic costs incurred by constraining the dsDNA at very high densities within the fixed volume of these phage capsids generate a significant internal pressure [1-6]. This pressure is considered to provide the force initially driving genome injection into the cell, while DNA-binding proteins, DNA condensation or enzymes may aid during the final stages of the transfer [7-12].

A common trait for all tailed dsDNA phages is that their proteinaceous DNA injecting machineries are always prepared or completed prior to the time of infection (Fig. 1A). Likewise, for this group the DNA exits the capsid through the tail. For members of the Myoviridae family, energy stored in the conformation of the proteins of their long contractile phage tails is critical for tail tube penetration of the outer membrane and the peptidoglycan cell wall, and thus to reach the inner cell membrane [13] (Fig 1A, middle). For noncontractile tails, neither the short (Podoviridae family, Fig 1A, left) or long tails (Siphoviridae family, Fig. $1 A$, right) are known to provide energy for the infection process $[14,15]$.

In contrast to the wealth of experimental and theoretical information that has been acquired about the genome entry of tailed dsDNA bacteriophages [16] (Fig. 1A), very little is known about this process for viruses with an internal membrane vesicle (Fig. $1 B$, top). 
A
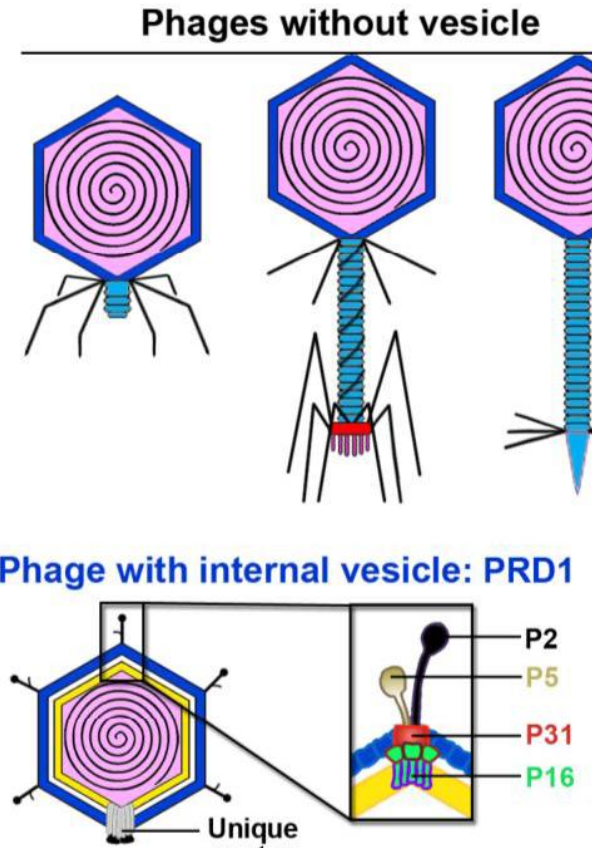

B Phage with internal vesicle: PRD1
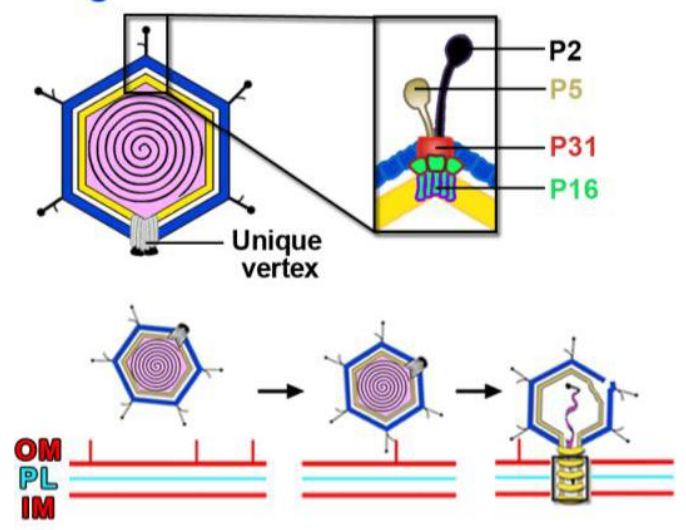

Fig. 1. Morphologies of various phage families.

(A) Schematic representation of the three families of tailed dsDNA bacteriophages whose genome ejection process has been previously studied: (left) the Podoviridae (e.g., phage T7, Q29); (center) the Myoviridae family (e.g., phage T4); (right) the Siphoviridae family (e.g., phage $\lambda$, SPP1); (blue) proteinaceous capsid and tail; (light magenta) viral DNA.

(B) (above) Schematic representation of

internal membrane-containing bacteriophage PRD1; (yellow) the internal membrane vesicle; (black) vertex proteins; (grey) the unique vertex; (inset) the proteins comprising the eleven host recognition vertices. (below) Cartoon of the PRD1 infection process adapted from [17] with outer membrane (OM), peptidoglycan layer (PL) and inner membrane (IM).

Membrane-containing bacteriophage PRD1 (Tectiviridae family) is the model system for this viral class. It infects gram-negative enterobacteria such as Escherichia coli and Salmonella enterica. The phage membrane is constituted of a 50:50 mixture of phage-encoded proteins and host-derived lipids. The lipid components are primarily phosphatidylglycerol (PG) in the outer leaflet and phosphatidylethanolamine (PE) in the inner one, with PG being negatively charged and PE uncharged at $\mathrm{pH} 7$ [18, 19]. Several phage-encoded, membrane-associated 
proteins have active roles during DNA delivery [20]. Determination of the structure of the PRD1 packaging vertex recently revealed that this phage membrane also has an important role in genome packaging [21]. The $\sim 15 \mathrm{kbp}$ linear dsDNA PRD1 genome is packaged to a density comparable to the high density seen for bacteriophage $\varphi 29$ [19]. The resultant significant pressure has been suggested to power DNA ejection for PRD1 [19].

Each host recognition vertex complex is composed of penton protein P31, spike protein P5, receptor recognition protein $\mathrm{P} 2$, and membrane protein $\mathrm{P} 16$ (Fig. $1 B$ top, inset). Binding of this complex to PRD1's cellular receptor elicits structural changes at this vertex. Most probably the loss of the interactions between the P16 proteins and the membrane vesicle loosens the vertex complex. The consequent changes to the environmental conditions experienced by the membrane inside the capsid are thought to initiate the remodeling of the internal icosahedral membrane into a tubular structure protruding from the unique vertex [17]. In this model, this self-assembling proteolipidic tube conducts the phage genome through the cell envelope of the gram-negative bacterial host and into the cytoplasm (Fig. $1 B$, below). We hypothesized that the free-energy cost to initiate this process is paid by the changes in osmotic pressure [17]. Although the precise energetics of this mechanism remain unsolved, we previously showed that tube formation is dependent on the physicochemical properties of the phage vesicle and its constituent membrane proteins, but is independent of the presence of the packaged DNA [17]. The formation of the tube is not examined further in this work.

We considered that the internal membrane of PRD1 might have an additional important function during ejection of the dsDNA because it becomes gradually smaller as the linear dsDNA molecule passes into the cell [17] (Fig. 2A-C). Here we focus on analysis of the 
potential role of the membrane vesicle on DNA ejection dynamics in PRD1 using the available biochemical and structural information on wild-type PRD1 and on procapsids devoid of DNA produced by sus1 amber mutant phage. To this end, we developed a semiempirical approach that incorporates the known physical properties of the membrane into a model for phage genome ejection dynamics. We propose that the presence and subsequent deflation of the internal membrane vesicle in PRD1 may help to sustain the significant driving force required to overcome the internal pressure of the bacterial host and may also facilitate the early stages of DNA ejection. 


\section{Materials and Methods}

\subsection{Production of purified PRD1 particles}

Wild-type PRD1 (PRD1) was propagated in non-suppressor host Salmonella enterica Typhimurium LT2 DS88 and purified by polyethylene glycol-NaCl precipitation, rate zonal centrifugation, and equilibrium centrifugation in sucrose [22]. Lastly, the phage sample was concentrated by differential centrifugation and dissolved in a buffer containing $20 \mathrm{mM}$ potassium phosphate $(\mathrm{pH} 7.2)$ and $1 \mathrm{mM} \mathrm{MgCl}$.

\subsection{Segmentation of tomograms and structural analysis}

Cryo-electron tomography (cryo-ET) data were collected in-house on a JEM-2200FS/CR, field emission gun (FEG) electron microscope operating at $200 \mathrm{kV}$ equipped with an omega in-column energy filter as previously described [17]. Briefly, single-axis tilt-series over a tilt range of \pm 64 with $1.5^{\circ}$ increments of wt PRD1 specimen were recorded using an Ultrascan $40004 \mathrm{~K} \times 4 \mathrm{~K}$ camera (Gatan Inc.). Serial EM software [23] was used for semiautomatic data acquisition (total dose per serie $90-100$ electrons $/ \AA^{2}$ ) at underfocus values ranging from 5 to $8 \mu \mathrm{m}$ and magnification of 30,000 and 25,000 leading respectively to a pixel size of $0.76 \mathrm{~nm}$ and $0.88 \mathrm{~nm}$ at the specimen after a binning of 2 . Alignment using $10 \mathrm{~nm}$ gold particles as fiducial markers and $3 \mathrm{D}$ reconstruction of the tilted series was performed in IMOD and/or Tomo3D software [24]. 3D reconstruction was carried out by weight back-projection or SIRT. Several tomograms were reconstructed from which we initially extracted 1,207 PRD1 particles with no tube and 251 volumes corresponding to PRD1 particles with a tube and tubes alone (see Table S1). Prior to further analysis sub-volumes with smaller voxel size were interpolated to a voxel of $0.88 \times 0.88 \times 0.88 \mathrm{~nm}^{3}$. For the current study we focused on the smaller subset and within the PRD1 particles with a tube we selected only those for which the viral membrane could be discerned as intact across the majority of reconstructed volume 
sections. A total of 12 PRD1 subvolumes possibly depicting different putative ejection stages were selected from this dataset (Table S1). TOMOAND [25] was used to denoise the individual volumes prior to segmentation in AMIRA 5.0 (http://www.fei.com/software/amira-

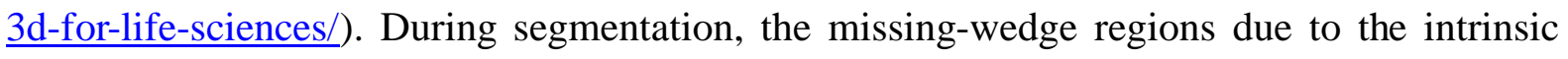
limitation of the tilt angle in the tomographic data collection were user-modeled as spherical domes in AMIRA. Volumes were then estimated from the segmented regions. Chimera and ImageJ software were also used for molecular visualization and analysis [26, 27].

The radial average profiles were calculated from the electron density maps of PRD1 (EMD1011) and procapsid (EMD-1013) particles using XMIPP v3.1 [28]. The membrane radius of the procapsid was estimated taking into account the distance from the center of the virus to the membrane layers along the three-fold axis.

\section{Results}

\subsection{Morphology of the phage internal membrane during DNA ejection}

Cryo-ET of individual PRD1 virions, each with a tube, had previously provided threedimensional (3D) snapshots of DNA ejection at different stages [17]. DNA ejection is a continuous process and currently there is no known mechanism for synchronous triggering of PRD1 tube formation in vitro. As a consequence, the visualized PRD1 particles constitute a heterogeneous ensemble of intact virions and virions with a tube. 
The volumes enclosed by the membrane vesicles were estimated for twelve 3D reconstructed PRD1 particles, each with a tube. This set was selected based on the visual completeness of

A

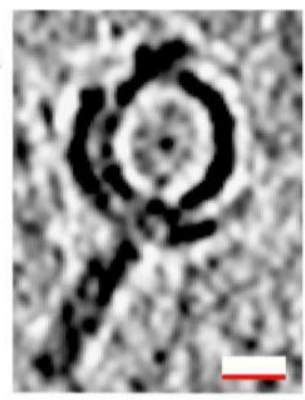

B
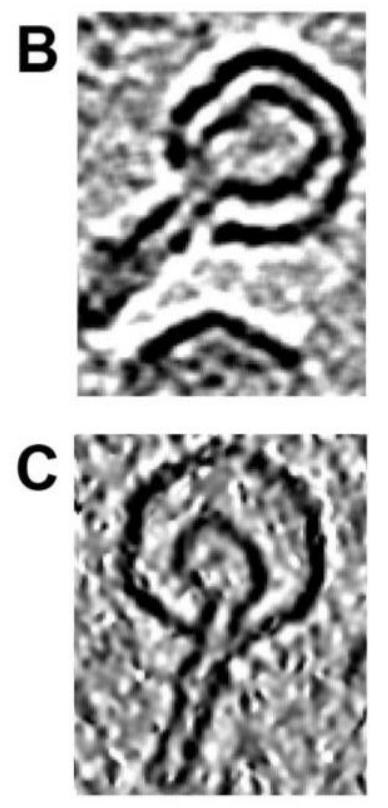
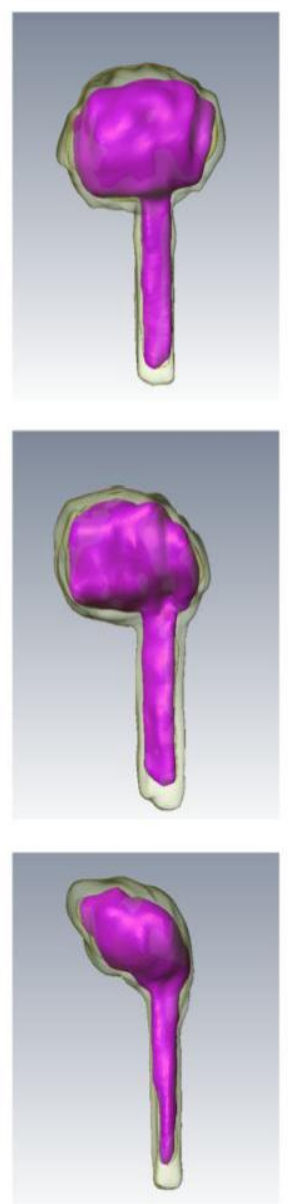

information while also taking into account the missing wedge effect that is unavoidable in tomography (see Materials and Methods). Using this approach, the twelve analyzed PRD1 particles with a tube could be assigned to three classes corresponding to three stages in the DNA ejection process. Although this set cannot represent all possible steps in this DNA ejection (framing a continuum of vesicle morphologies escapes the current experimental setup), they might depict consecutive stages in DNA delivery (Fig. $2 A$ C) [17].

Fig. 2. Morphologies of PRD1 virions during DNA ejection.

(A) to $(C)$ Central slice $(0.88 \mathrm{~nm}$ thickness) of the denoised 3D tomogram of PRD1 with a protruding tube (left) and segmented vesicle (right) at consecutive stages of DNA ejection. (right) The enclosed volume purportedly occupied by the viral genome is colored in magenta, and that by the segmented vesicle in semi-transparent yellow (see also Fig. S1); scale-bar 20 $\mathrm{nm}$. 
Thus, we defined the initial ejection phase to correspond to a vesicle volume $\left(\left\langle V_{\text {initial }}\right\rangle \cong\right.$ $2.7 \times 10^{4} \mathrm{~nm}^{3}, \sigma_{\text {Vini }} \cong 1.8 \times 10^{3} \mathrm{~nm}^{3}, \mathrm{n}=6$; see Fig. $\mathrm{S} 1$ for the set of particles used) that is greater than $60 \%$ of the pre-infection vesicle volume $\left[\mathrm{V}_{\text {Pre }} \cong 4.0 \times 10^{4} \mathrm{~nm}^{3}\right.$; calculated from the crystal structure [19]] (Fig. 2A). Likewise, we defined an intermediate stage to correspond to vesicle volumes between $30 \%$ and $60 \%$ of the pre-infection volume $\left(\left\langle\mathrm{V}_{\text {intermediate }}\right\rangle \cong 1.7 \times 10^{4}\right.$ $\mathrm{nm}^{3}, \sigma_{\text {Vint }} \cong 6.2 \times 10^{3} \mathrm{~nm}^{3}, \mathrm{n}=2$ ) (Figs $2 B$ and $\mathrm{S} 1$ ). The final stage corresponds to a strikingly altered vesicle morphology that now resembles a 'map pin' enclosing less than $30 \%$ of the initial volume $\left(<\mathrm{V}_{\text {final }}>\cong 8.7 \times 10^{3} \mathrm{~nm}^{3}, \sigma_{\mathrm{Vfin}}=\cong 2.9 \times 10^{3} \mathrm{~nm}^{3}, \mathrm{n}=4\right)($ Figs $2 C$ and $\mathrm{S} 1)$. These three vesicle morphologies have also been captured by cellular cryo-ET analysis of PRD1 infected E. coli cells [17].

\subsection{Estimation of the internal pressure on the membrane exerted by the packaged DNA and} the forces driving the DNA ejection

The PRD1 genome enclosed within the membrane vesicle is assumed to be wound approximately into a spool-like configuration [19]. Therefore, we used the inverse-spool model $[1,3,29,30]$ to estimate the theoretical internal pressure exerted by the phage DNA. This model was first introduced by Kindt and colleagues [1] and Tzlil and co-workers [30], building up on previous ideas by Riemer and Bloomfield [31] and Odijk [32] among others. Here, we will use the specific mathematical framework employed by Purohit and co-workers $[3,29]$, more suitable for the non-condensing, purely repulsive regime of interactions expected inside PRD1. In this model, the total free energy of confined DNA $G_{\text {tot }}$ is assumed to consist of two main contributions: $(i)$ the elastic bending energy $G_{b e n d}$, due to the fact that a length $L$ of DNA is bent to a radius that is less than the $50 \mathrm{~nm}$ persistence length of dsDNA, and (ii) the interaction energy $G_{i n t}$, accounting mainly for the electrostatic, entropic, and 
hydration interactions among neighboring DNA strands that are separated by an average inter-strand distance $d_{S}$ :

$$
G_{\text {tot }}\left(L, d_{s}\right)=G_{\text {bend }}+G_{\text {int }}
$$

The elastic contribution

$$
G_{\text {bend }}(L)=-\frac{4 \pi \xi_{p} k_{B} T}{\sqrt{3} d_{s}^{2}}\left(\sqrt{R_{\text {out }}^{2}-R^{2}}+R_{\text {out }} \ln \left(\frac{R_{\text {out }}-\sqrt{R_{\text {out }}^{2}-R^{2}}}{R}\right)\right)
$$

is calculated by assuming that the DNA inside the capsid is arranged in an inverse spool configuration of external radius $R_{\text {out }}$ and internal radius $R$, where the strands are packed in a hexagonal array with an average spacing $d_{s}$. In the previous expression $\xi_{p}$ is the persistence length of dsDNA $(50 \mathrm{~nm}), k_{B}$ is Boltzmann's constant, $T$ is the temperature. This elastic contribution usually accounts for $10 \%$ of the total energy [33].

The total length $L$ of the spooled dsDNA is then

$$
L=\frac{8 \pi}{3 \sqrt{3} d_{s}^{2}}\left(R_{o u t}^{2}-R^{2}\right)^{3 / 2}
$$

The interaction energy $G_{i n t}$ is evaluated from osmotic stress experiments at the proper conditions using the empirical expression

$$
G_{\text {int }}=\sqrt{3} F_{0} L\left(c^{2}+c d_{s}\right) e^{-d_{s} / c}
$$


For a typical buffer (50 mM Tris-HCl, $\mathrm{pH} 7.8,100 \mathrm{mM} \mathrm{Nacl}$ and $10 \mathrm{mM} \mathrm{MgCl}_{2}$ ) [34], the decay length represented by $c$ is $0.30 \pm 0.01 \mathrm{~nm}$ and the strength of interactions is characterized as $F_{0}=(3 \pm 1) 10^{4} \mathrm{pN} / \mathrm{nm}^{2}$. Note that, at these conditions, the interactions between dsDNA strands inside the virus are purely repulsive. As in Purohit et al [3, 29], we omit the surface energy terms associated to the missing neighbors on the surface of the spool and the interactions of the dsDNA with the interior of the capsid or the inner membrane (the inner leaflet in PRD1 is mainly composed of zwitterionic PE).

Using these bending and interaction terms, the optimal energy and DNA spacing are then obtained by minimizing the total energy $G_{\text {tot }}\left(L, d_{s}\right)$ with respect to $d_{s}$ for a given external radius $R_{\text {out }}$ and total genome length $L$.

The internal pressure $(\mathrm{P})$ and the force driving the DNA ejection $(\mathrm{F})$ can then be obtained from $G_{t o t}\left(L, d_{s}\right)$ using their standard thermodynamic definitions:

$P=-\left(\frac{\partial G_{t o t}}{\partial V}\right)=-\frac{1}{4 \pi R_{\text {out }}^{2}}\left(\frac{\partial G_{\text {tot }}}{\partial R_{\text {out }}}\right)$

$F=-\left(\frac{\partial G_{t o t}}{\partial L}\right)$

where $R_{\text {out }}$ is defined as the outer radius of the spool of packaged DNA. Therefore, the pressure and translocation forces can be calculated given the total length of the PRD1 genome and the volume in which it is enclosed. 
Let us now estimate the values of the pressure and translocation forces of dsDNA in PRD1 for two simple cases: a PRD1 capsid with a static, non-deflating membrane, and a PRD1 capsid without a membrane.

For simplicity, in our calculations the icosahedral volumes $V_{i c o}$ of the mature PRD1 capsid and membrane vesicle are modeled as effective spheres with radius $R_{\text {eff }}$ that enclose an equal volume, i.e $\frac{4 \pi}{3} R_{\text {eff }}^{3}=V_{i c o}$.

To calculate the icosahedral volume enclosed by the membrane, the internal radius of the membrane vesicle $r_{i}$ was estimated to be $20 \mathrm{~nm}$, i.e., half the distance between icosahedral membrane facets based on the PRD1 X-ray structure [19]. If $\mathrm{r}_{\mathrm{i}}=20 \mathrm{~nm}$, then $V_{\text {ico }} \cong 40,432$ $\mathrm{nm}^{3}$. This same volume would be enclosed by a spherical vesicle with an effective radius $R_{\text {eff }}=21.3 \mathrm{~nm}$.

Lastly, $R_{\text {out }}^{\text {mem }}$ (the outer radius of the spooled DNA within this spherical vesicle of radius $R_{\text {eff }}$ ) is obtained by subtracting from this radius the $2 \mathrm{~nm}$ between the membrane and the outermost DNA layer (for a visual glossary see Fig. S2). Thus, initially $R_{o u t}^{m e m}=19.3 \mathrm{~nm}$.

Considering the phage membrane to be a rigid structure containing the 14,927 bp genome, the internal pressure exercised by the PRD1 packaged DNA (Eq. 5) before infection is estimated to be $\sim 52 \mathrm{~atm}$ (Fig. 3A, blue line). This is consistent with a previous estimate of $\sim 45 \mathrm{~atm}$ [19]. Moreover, the calculated DNA strand spacing $\left(d_{s}\right)$ agrees with the experimentally observed value of $2.6 \mathrm{~nm}$ (Fig. 3B, blue line) [19]. Based on this model, a maximum force of $39.9 \mathrm{pN}$ would drive the initial DNA ejection when $100 \%$ of the DNA is within the virion (Fig. $3 C$, blue line). 

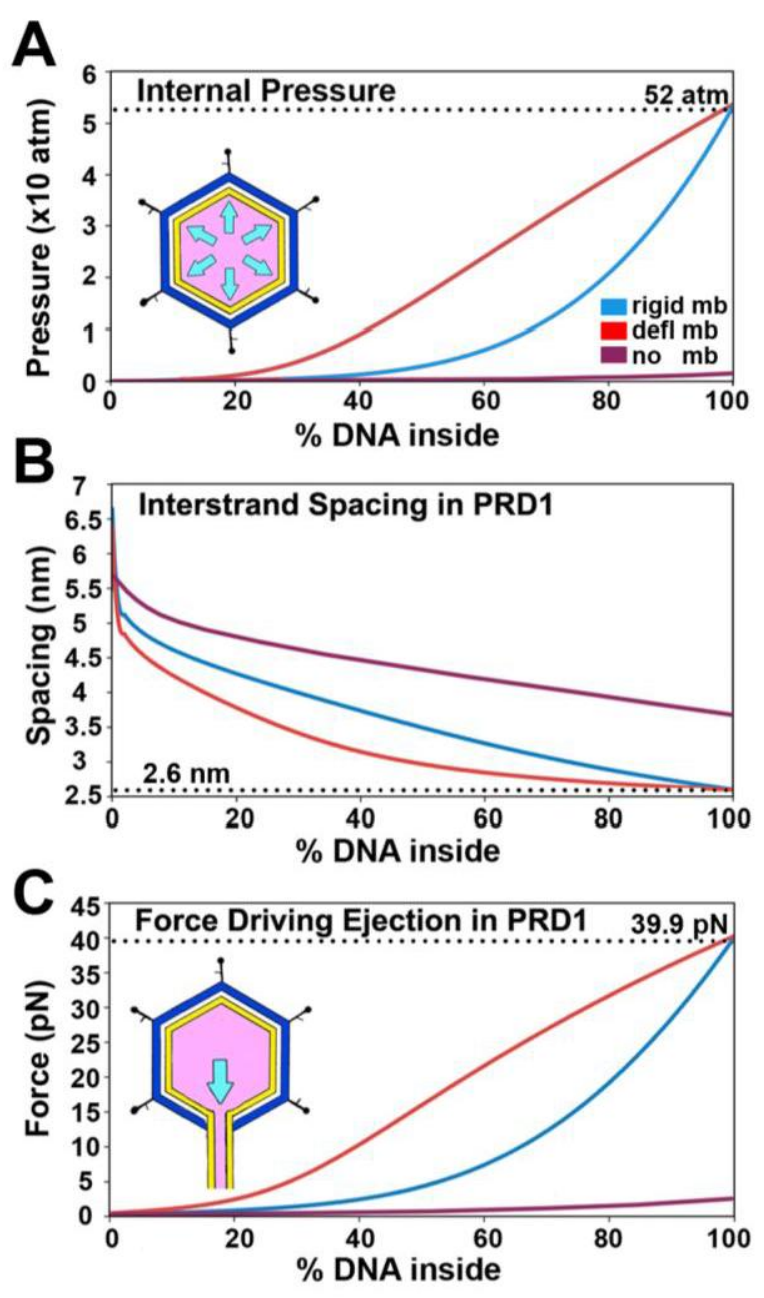

Fig. 3. Comparative pressure and force analyses for PRD1 virions modeled during DNA ejection.

(A) Pressure inside a PRD1 virion containing, in addition to the packaged DNA, a rigid membrane (blue line), an elastic, deflating membrane (red line), and no membrane (purple line), as a function of the percent of the DNA genome inside. (inset)

(B) Inter-strand spacing in PRD1 virions with a rigid membrane, an elastic, deflating membrane, or no membrane as a function of the percent of the DNA genome inside

(color coded as in A).

(C) Force driving DNA ejection from PRD1 virions with a rigid membrane, an elastic, deflating membrane, or no membrane as a function of the percent of the DNA genome inside (color coded as in A). (inset) Light blue arrow indicates directionality of the force driving DNA ejection.

To assess the impact of the phage membrane on the internal pressure, DNA strand spacing, and potential ejection force, the above calculations were repeated considering the DNA confinement generated by a protein capsid without a membrane. In this case the genome would be inside an icosahedral capsid with an internal radius of $25.5 \mathrm{~nm}$, corresponding to a 
effective sphere of radius $27.2 \mathrm{~nm}$. Again deducting the $2 \mathrm{~nm}$ distance separating the shell from the outermost DNA layer yields an $R_{\text {out }}^{\text {capsid }}=25.2 \mathrm{~nm}$. This increased volume now available inside the capsid would allow the DNA inter-strand spacing $\left(d_{s}\right)$ to increase from $2.6 \mathrm{~nm}$ to $3.7 \mathrm{~nm}$ (Fig. $3 B$, purple line). Using $R_{\text {out }}^{\text {capsid }}=25.2 \mathrm{~nm}$ in Eq. 5 drastically reduces the estimated internal pressure to $\sim 1.5$ atm, a value similar to or even lower than the turgor pressure within the host bacterial cell [7, 35] (Fig. 3A, purple line). The corresponding maximum force driving the ejection would be reduced to only $\sim 2.5 \mathrm{pN}$ (Fig. $3 C$, purple line).

Therefore, a PRD1 virion with the same genome length but without the membrane would be unable to successfully inject its DNA into a host cell possessing significant turgor pressure. The mere physical presence of the membrane is essential for reducing the space available in the capsid for the packaged genome (i.e., for increasing the degree of confinement of the DNA) and thereby generating greater pressure and translocation force.

\subsection{A model for PRD1 membrane-assisted DNA ejection based on membrane stress and tension as derived from PRD1 virion and procapsid analysis}

The observed remodeling of the internal membrane vesicle in response to environmental changes [17] shows that the membrane is not a rigid shell. To evaluate a possible contribution of the viral membrane to DNA ejection beyond its role as rigid container, we postulated that the remodeled membrane would retain its elastic properties. Due to the challenges in modeling such a viral proteo-lipidic system, we used the structural theory of thin shells and membranes originally applied to biological membranes by Helfrich [36]. In this approximation, the lipid bilayer (even with the presence of embedded membrane proteins) is treated as a continuum and it is modeled as a thin spherical shell of uniform thickness $h$ and 
internal radius $R$. Because in our case $R \gg h$, we can apply the linear membrane or thin shell theory (TST) [37, 38].

In the TST model, the change of radius $\Delta \mathrm{R}$, change of thickness $\Delta \mathrm{h}$, and the lateral stress $\sigma_{\text {lat }}$ of a spherical shell of initial thickness $h_{0}$ and radius $R_{0}$ due to the difference between its internal and external pressure $\mathrm{p}$ are given by:

$\Delta \mathrm{R}=\mathrm{R}-\mathrm{R}_{0}=\frac{1-\sigma}{\mathrm{Y}} \frac{\mathrm{R}_{0}^{2}}{2 h_{0}} \mathrm{p}$

$\Delta \mathrm{h}=\frac{-1}{\mathrm{Y}} \mathrm{R}_{0} \sigma \mathrm{p}$

$\sigma_{\text {lat }}=\frac{p R_{0}}{2 h_{0}}$

where $\sigma$ is the Poisson ratio (describing the relative expansion in the transverse directions when a material is compressed longitudinally) and $\mathrm{Y}$ is the Young's modulus (defined as the ratio between force per unit area needed to stretch/compress a given material and the resulting deformation per unit length). Typical values of the Poisson ratio for a membrane are in the range $0.3 \leq \sigma \leq 0.5$ (incompressible) [39].

TST is equivalent to the standard membrane elasticity description of lipid bilayers with a bending elasticity $\kappa_{b}=\frac{Y h^{3}}{24\left(1-\sigma^{2}\right)}$ and an extensional stiffness (or two-dimensional stretching modulus) $\kappa_{s}=\frac{Y h}{1-\sigma^{2}}$. For a simple homogeneous expansion of a spherical vesicle, this yields a stretching energy $E_{\text {stretch }} \propto \kappa_{s}\left(\frac{\Delta R}{R}\right)^{2}$ and a bending energy per unit area $E_{\text {bending }} \propto$ 
$\kappa_{b}\left(\frac{\Delta R}{R^{2}}\right)^{2}$ (34). The ratio between these two contributions, i.e., $\frac{E_{\text {stretch }}}{E_{\text {bending }}} \propto\left(\frac{R}{h}\right)^{2}$, is quite large for thin membranes. Accordingly, when this spherical vesicle is subjected to a significant internal pressure, stretching dominates over bending to such an extent that the latter can be neglected. The TST model is essentially assuming that the internal pressure stretches the membrane and generates a lateral tension given by $T=\sigma_{\text {lat }} h_{0}=\frac{\mathrm{pR}_{0}}{2}$, following the usual Young-Laplace equation.

Membrane stress values were then derived by comparing membrane vesicles in the procapsid (devoid of DNA) and in the mature PRD1 virion using their corresponding 3D cryo-EM reconstructions obtained using precisely the same experimental and processing procedures [40] (Fig. 4, centre). Moreover as the membrane vesicles of both procapsid and PRD1 virion contain the same set of membrane proteins, only the membrane vesicle with the packaged genome was considered as being under stress. This simplification allows us to pinpoint the possible contribution of an 'elastic' vesicle (rather than a rigid one) to the DNA ejection force.

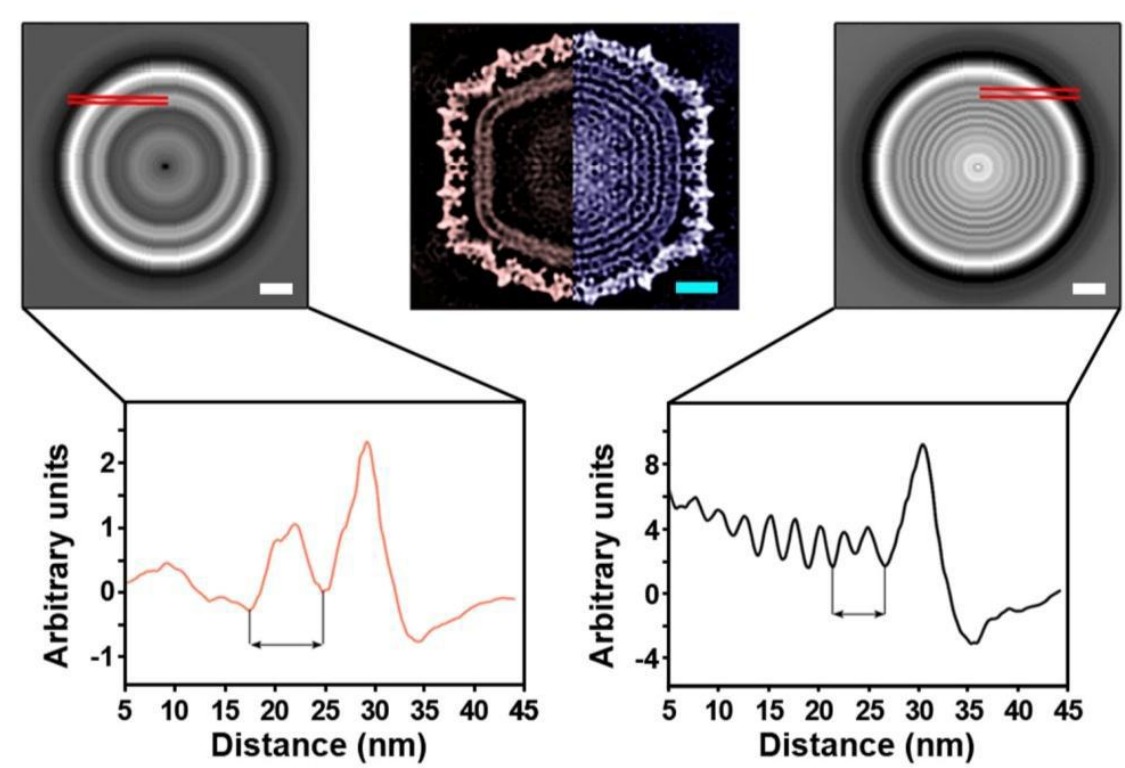

Fig. 4. Comparison of vesicle structure in PRD1 virions and procapsids. 
(Center) Composite image with central sections of the 3D cryo-EM reconstructions of the procapsid in pink (EMD-1013) and PRD1 virion in blue (EMD-1011); (cyan) scale bar 10 $\mathrm{nm}$. Flanking the central image are the calculated radial average images of the maps for (left) the procapsid and (right) the virion; (red lines) the outer and inner layer of the membrane vesicle; (white) scale bars $10 \mathrm{~nm}$. (insets below) the corresponding radial averaged profiles with estimates of vesicle bilayer total thickness (black double-headed arrow).

The membrane vesicle is also icosahedral in the procapsid [40] (a non-icosahedral feature would blur during the 60 -fold averaging procedure). Its internal radius estimated along the icosahedral three-fold axis of symmetry is $r_{i}=16.8 \mathrm{~nm}$. This is $3.2 \mathrm{~nm}$ less than seen in the mature virion (in pink Fig. 4, center), and is equivalent to an effective spherical radius $R_{0}^{m e m}=17.9 \mathrm{~nm}$. Since no phage genome is present, we assume that this radius corresponds to that of a stress-free spherical membrane vesicle. Conversely, the effective radius of the membrane in the virion would correspond to the membrane expanded by the pressure exerted by the packaged genetic material (in blue Fig. 4, center) $[19,40,41]$.

The total membrane thickness was estimated using the electron density radial profiles derived from the procapsid and virion density maps (Fig. 4, left and right). The distance between the minima flanking the lipid head groups is $\sim 7.4 \mathrm{~nm}$ for the procapsid and $\sim 5.5 \mathrm{~nm}$ for the virion (black double-headed arrow in Fig. 4 insets). 
A
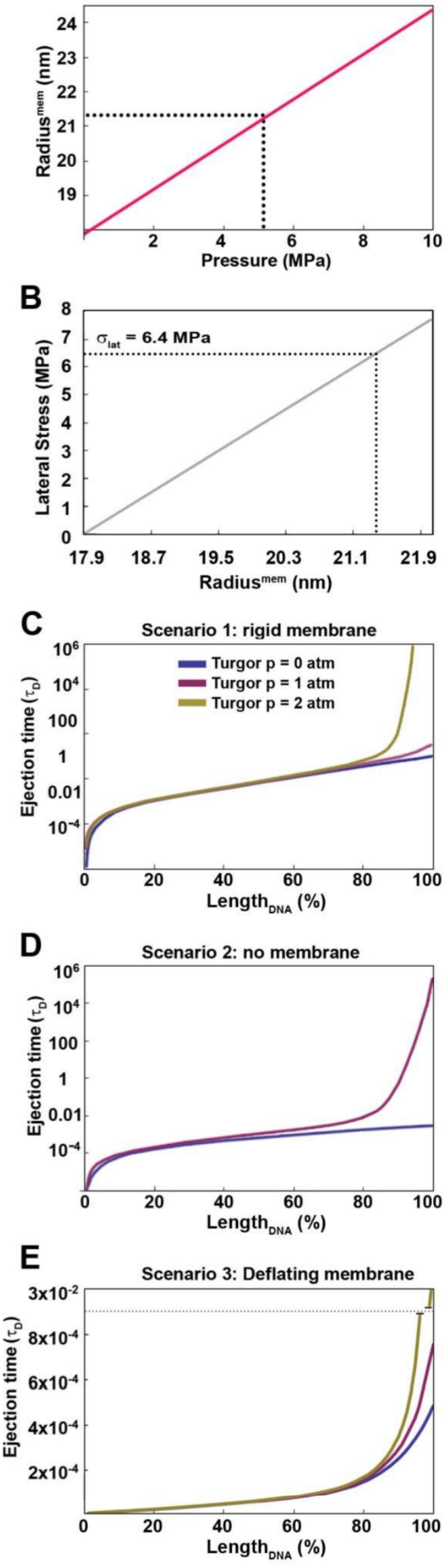

Although the value of the Young's modulus for the membrane is not known with certainty, we used a value of $\mathrm{Y}=20 \mathrm{MPa}$ and a Poisson ratio $\sigma=0.4$ based on previous studies [42]. This also matches the effective compression observed in the membrane for the internal pressure due to the confined DNA.

Using a membrane radius $R_{0}^{m e m}=17.9 \mathrm{~nm}$ and thickness of $h_{0}=7.4 \mathrm{~nm}$ for the unstressed membrane, a value of $20 \mathrm{MPa}$ for Young's modulus, and a Poisson ratio of 0.4 , one can estimate (from Eq. 7) the value for the internal radius of the spherical membrane generated by a given pressure (Fig. 5A).

Fig. 5. Impact of three distinct membrane models on DNA ejection dynamics.

(A) PRD1 membrane vesicle internal pressure as a function of the membrane radius. A pressure of 52 atm corresponds to a $\mathrm{R}^{\mathrm{mem}} \sim 21.3 \mathrm{~nm}$.

(B) Lateral stress $\left(\sigma_{\text {lat }}\right)$ as a function of the membrane radius. This lateral stress is $6.4 \mathrm{MPa}$ for the virion and is assumed to be 0 for the procapsid devoid of DNA.

(C) For PRD1 with a rigid membrane: ejection time 
required for a given percentage of the DNA molecule to enter the host expressed in units of the free diffusion time $\tau_{\mathrm{D}}$ for three cellular turgor pressures: (blue) $\mathrm{p}_{\mathrm{int}}=0$ atm; (dark red) $\mathrm{p}_{\mathrm{int}}=1 \mathrm{~atm} ;($ green line $) \mathrm{p}_{\mathrm{int}}=2 \mathrm{~atm}$.

(D) For PRD1 without a membrane and for two turgor pressures, shown and color coded as in $(C)$.

(E) For PRD1 with an elastic, deflating membrane and for three turgor pressures, shown and color coded as in $(C)$.

At the pressure of $52 \mathrm{~atm}$ (previously estimated assuming the vesicle to be a rigid container; Fig. $3 A$ ) the inner membrane radius would correspond to $\mathrm{R}^{\mathrm{mem}}$ of about $21.3 \mathrm{~nm}$. Also, the change in the membrane thickness at this pressure, $\Delta \mathrm{h}=-1.9 \mathrm{~nm}$, is similar to the reduction in effective membrane thickness between the procapsid and virion as estimated from Fig. 4, insets. Furthermore, the lateral stress $\left(\sigma_{\text {lat }}\right)$ in the membrane, given by Eq. 9 and plotted in Fig. $5 B$ reaches a value of $6.4 \mathrm{MPa}$ for the virion and is, by definition, zero for the procapsid. These estimates would differ if other values of $\sigma$ and $\mathrm{Y}$ were chosen. Nevertheless, the significant conclusion is that, given the approximation of the thin-shell-theory and accepted values for the elastic properties of the membrane, the phage membrane within the capsid responds to, and is influenced by, the pressure of the confined DNA.

Within this framework one can envision a new, but simple, model for membrane-assisted DNA ejection. Rather than simplistically considering the vesicle as a rigid container, we can incorporate the interplay between an elastic membrane and the decreasing pressure exerted by the confined DNA. Here we assume that as ejection proceeds, the pressure required to keep 
the membrane vesicle inflated to a radius $R_{m}$ is the same as the pressure generated by the dsDNA confined in a spherical volume of radius $R_{m}$.

The pressure $p\left(R_{m}, L\right)$ due to a length $L$ of DNA confined inside the membrane with internal radius $R_{m}$, can be calculated from Eq. 5, and likewise the pressure required to keep the membrane vesicle inflated with an internal radius $R_{m}$ is given by Eq. 7 . Equating these two pressures yields an expression for $R_{m}(L)$ that indicates how much DNA is inside the membrane with a radius $R_{m}$. From this expression, we determined the DNA pressure, interstrand spacing, and ejection force throughout the ejection as plotted in Fig. $3 A-C$ (red lines).

Based on this, $R_{m}(L)$ can provide some insights on how much DNA is expected to still remain inside the membrane at the various stages of DNA ejection (Fig. $2 A-C$ ). During the initial ejection stage, when the average volume enclosed by the spherical part of the membrane is $\mathrm{V}_{\text {ico }}=2.5 \times 10^{4} \mathrm{~nm}^{3}$ and $R_{m}=18 \mathrm{~nm}$, the model predicts that $\sim 25 \%$ of the genome remains inside the capsid.

In the consecutive stages (Fig. $2 B-C$ ), the volume enclosed by the spherical part of the membrane would be less than that of the vesicle in the Sus1 procapsid that is devoid of DNA. This suggests that some proteo-lipidic material could be lost from the membrane (plausibly from the distal tip of the tube that is now open into the cytoplasm) during DNA ejection, possibly increasing the ejection force available during later stages of the ejection process. In this light, our simple initial estimates can be considered to provide a conservative estimate of the active contribution of the membrane during genome ejection. 
3.4 Effects of an elastic membrane vesicle on the internal pressure and ejection times of the confined PRD1 genome

To evaluate the impact of the presence and deflation of the membrane on the in vivo phage DNA delivery process, we estimated the ejection times for different values of the internal bacterial turgor pressure.

The dynamics of DNA ejection can be described in terms of a Fokker-Planck equation for $P(x, t)$, where the probability that a given length $x$ of DNA has entered inside the host cell at time $t[9,10]$ is:

$\frac{\partial P(x, t)}{\partial t}=\frac{\partial}{\partial x}\left(D(x) \frac{\partial P(x, t)}{\partial x}+\frac{D(x)}{k_{B} T} \frac{\partial W(x)}{\partial x} P(x, t)\right)$

where $D(x)$ is the diffusion coefficient (which may depend on $x$ ), and $W(x)$ is the free energy of the system. Further, the effective force driving DNA ejection is then $F(x)=$ $-\left(\frac{\partial W}{\partial x}\right)$

In this framework, the time $\tau(x)$ required for a given DNA length $x$ to enter the cell is given by the Mean First Passage Time (MFPT):

$$
\tau(x)=\int_{0}^{x} d y \frac{1}{D(y)} \exp \left[\frac{W(y)}{k T}\right] \int_{0}^{y} d z \exp \left[\frac{-W(z)}{k T}\right]
$$


Assuming a constant diffusion coefficient, $D(x)=D$ and the absence of any driving force, the MFPT for the entrance of an entire DNA chain of length $L$ becomes the usual diffusion time $\tau_{d}=\frac{L^{2}}{2 D}$. That time will be used as our time unit in the following results.

When the DNA ejection occurs against the internal turgor pressure $p_{\text {int }}$ of the host cell, the free energy of the system has to be adjusted to correct for the pressure-volume work required to inject a given length $x$ of DNA into the host. In principle, there is another contribution that we should take into account, related to the fact that the osmotic pressure inside the bacterial host promotes DNA condensation [7, 8], which in turn would enhance the extent of ejection. However, it is not easy to quantify accurately this contribution, since DNA condensation strongly depends on the condensing agent or conditions, and surface effects. An estimate by Marion and Siber [7] suggests that this condensation effect can compensate between $0.5 \mathrm{~atm}$ up to about 4 atm of osmotic pressure. But, still in most cases the dominant effect of osmotic pressure is to resist DNA injection, as shown for instance in the experiments by Jeembaeva et al [8], where at 4 atm of osmotic pressure only $60 \%$ of lambda DNA was ejected, even allowing for its condensation. Since the quantification of the right condensing conditions inside the host is challenging, we opted to not include this effect explicitly in the calculations. In any case, this effect can be partially incorporate by considering $p_{\text {int }}$. as the effective turgor pressure resisting the entrance of dsDNA. Therefore:

$W(x)=G_{t o t}(x)+\pi x r_{D N A}^{2} p_{\text {int }}$

The values reported for the turgor pressure of $E$. coli are between $0.5-5$ atm [43, 44]. Therefore, our calculations were performed for turgor pressures of $0,1,2,3$, and 5 atm, while using the force vs length values reported above (Fig. 3C). Based on these data and 
assumptions, we evaluated three different scenarios: a rigid membrane, no membrane, and an elastic, deflating membrane.

For a rigid membrane, the time required for complete DNA ejection was calculated for various turgor pressures. For $p_{\text {int }}=0$ atm, $\tau_{F}=1.0 \times 10^{-3} \tau_{d}$, i.e., three orders of magnitude faster than free diffusion. With $p_{\text {int }}=1 \mathrm{~atm}, \tau_{F}=3.1 \times 10^{-3} \tau_{d}$, i.e., three-fold slower than in the absence of turgor. With greater turgor pressures of 2,3 , and $5 \mathrm{~atm}$, the times are unrealistically long $\left(\tau_{F}=5.9 \times 10^{9} \tau_{d}, \tau_{F}=4.4 \times 10^{40} \tau_{d}\right.$, and $\tau_{F}=1.5 \times$ $10^{134} \tau_{d}$, respectively. Moreover, for 2,3 , and $5 \mathrm{~atm}$, only $87 \%, 78 \%$ and $65 \%$, respectively, of the genome can enter the host before the turgor pressure halts the ejection (Fig. $5 C$ ).

Without a membrane, the total injection time for $p_{\text {int }}=0$ atm would be $\tau_{F}=3 \times 10^{-3} \tau_{d}$, only three-fold slower than with the rigid membrane because the force, though much smaller, still speeds up the ejection significantly relative to free diffusion. However, the absence of a membrane changes injection times significantly when the turgor pressure of the bacterial cell is taken into account. Given even a minimal turgor pressure of $1 \mathrm{~atm}, \tau_{F}=2.510^{5} \tau_{d}$. For turgor pressures of 2,3 and 5 atm the entrance times are extraordinarily long $\left(\tau_{F}=\right.$ $7 \times 10^{68} \tau_{d}, \tau_{F}=10^{168} \tau_{d}$, and $\tau_{F}=10^{424} \tau_{d}$, respectively) since only $51 \%, 35 \%$ and $10 \%$ of the genome could enter the cell against the turgor pressure (Fig. 5D).

In the third scenario (Fig. $5 E$ ), as the DNA is ejected the elastic membrane deflates and thereby keeps the DNA constrained. As shown in Fig. 3B (red line), membrane deflation maintains closer inter-strand spacing while the DNA is exiting. Consequently, as the DNA leaves the capsid, both pressure (Fig. 3A, red-line) and force (Fig. 3C, red line) decrease more slowly relative to the rigid membrane model in which the membrane remains attached to the capsid wall. The times required for complete DNA entry in this elastic membrane scenario are 
for $p_{\text {int }}=0$ atm, $\tau_{F}=5 \times 10^{-4} \tau_{d}$; for $p_{\text {int }}=1 \mathrm{~atm}, \tau_{F}=7.6 \times 10^{-4} \tau_{d}$; and for $p_{\text {int }}=2$ atm, $\tau_{F}=3 \times 10^{-2} \tau_{d}$. Ejection times against higher turgor pressures become very long $\left(\tau_{F}=4.4 \times 10^{8} \tau_{d}\right.$ for $p_{\text {int }}=3$ atm and $\tau_{F}=1.110^{49} \tau_{d}$ for $p_{\text {int }}=5$ atm $)$ with only $92 \%$ and $83 \%$ of the DNA molecule, respectively, entering the cell before the turgor pressure blocks further ejection.

\section{Discussion}

The intrinsic complexity of a virion that includes the presence of an internal membrane vesicle makes the physical modeling of viral genome ejection an arduous task. It is not a coincidence that the modeling of viral DNA delivery had, so far, been contemplated only for bacteriophage virions that lack a membrane component [16]. We have endeavored to grasp the design and function of the internal membrane vesicle present within the capsid of some icosahedral viruses that also package their dsDNA to a high density using bacteriophage PRD1 as our prototype.

In this study, we (i) showed that PRD1 DNA ejection would not be viable in the absence of the internal lipid membrane, and (ii) formalized a novel model for membrane-facilitated DNA ejection for viruses that package their genome to high density within an internal membrane vesicle, thus creating high internal pressure. In this model, the essential elastic properties of the vesicle are introduced (Figs. 3-4). This novel, yet simple, model focuses on the steps that occur after the tube has formed and the flow of DNA into the host cell cytoplasm from the distal tube tip has begun. It relies on approximations that consider the membrane vesicle to be a continuum and the stress on the viral membrane to be derived only from the packaged genome (Fig. 4). In this simplification, the internal lipid bilayer is likened to an inflated balloon that was first stretched by the packaging of dsDNA to high pressure inside, and that subsequently pushes its contents out. This proposed framework suggests that the membrane 
tension aids the initial stage of DNA ejection (Figs. $2 A$ and $3 B-D$ ), a factor with clear impact on possible in vivo infection scenarios (Fig. 5C-E).

In addition, based on the constant tube-length [on average $\sim 50 \mathrm{~nm} ;(21)$ [17]] and partial integrity of the vesicle observed as the DNA exits (Fig. $2 A-C$ ), the membrane's contribution to the dynamics of DNA ejection might extend beyond the first stage (25\% of the genome remaining inside the vesicle; Fig. $2 A$ ) to the consecutive stages (Fig. $2 B-C$ ), as both the dynamic rearrangement of the proteo-lipidic material within the vesicle and the loss of some material from the tube tip are plausible. It is also possible that the PRD1 vesicle, expanded by the packaged DNA, could be initially stretched even further as a consequence of its interactions with the major capsid proteins and/or scaffolding proteins. Notably, proteinmembrane interactions have been proposed to be pivotal for the assembly of internal membrane-containing viruses [45-47]. It is conceivable, therefore, that even higher pressure could be exerted by the membrane on the DNA. If so, this would reduce the DNA strand interspacing during the ejection, thus generating even greater forces that could eject the DNA at a sufficient rate against even higher internal turgor pressures (Fig. 6).

Unfortunately verification of this proposed mechanism remains experimentally challenging. Currently, for example, the lack of a known PRD1 cellular receptor impedes osmotic suppression measurements such as the ones that have been elegantly performed on bacteriophages T5 and $\lambda[5,48]$. In the future, along with the search of a trigger for synchronous DNA ejection in PRD1, cryo-electron microscopy imaging of samples pretreated with detergent molecules capable to solubilize the lipid bilayer could also illuminate on the confinement capabilities of the PRD1 membrane vesicle on the pressurized dsDNA. 
The same treated particles (and after removal of detergent) could be also tested for cell attachment or/and infectivity experiments.

Thus, although our theoretical model offers only a partial, likely limited, and possibly not exclusive description of the viral DNA ejection mechanism for viruses with this virion architecture, it raises the possibility of an active role for the membrane vesicle. Even when the membrane contribution is insufficient to account for the ejection of all of the DNA, the remaining portion could be translocated by other mechanisms and forces, as has been suggested for other phages, e.g., by DNA condensation inside the host, hydration forces, and ratcheting in by proteins of the host cell [49]. These other mechanisms and forces must enter into play in the case of other membrane-containing viruses such as marine bacteriophage PM2 whose genome is supercoiled and with an entry mechanism different from that observed in PRD1 $[47,50]$ and giant viruses such as Mimivirus infecting amoeba. Mimivirus possesses an internal membraneous sac that contains a loosely packaged dsDNA genome $(\sim 1.2 \mathrm{Mb})$ and no pressure is expected. In this case infection seems to be initiated by the internalization of the entire virion, followed by the release of the genome by a star-shaped opening of the capsid with the membrane forming a conduit that fuses with the host membrane [51]. However, the large membrane containing PBCV-1 virus infecting algae has been suggested to also have a phage-like ejection mechanism [52]. Thus, the potential influence of the internal membrane vesicle on those viruses in the light of our work remains a fascinating but yet unexplored area of investigation.

\section{Conclusions}

Our analysis used the bacteriophage PRD1 as a model for viruses that include an internal membrane surrounding the densely-packaged dsDNA inside their icosahedral virions. The 
results reveal that the mere presence of the membrane helps to generate the significant initial pressure needed to counter the turgor pressure of the host during DNA translocation. In addition, membrane deflation might further increase the force driving the ejection dynamics during the early stages of genome delivery. Depending on the turgor pressure of the bacterial host, this extra force could be sufficient to ensure fast and efficient injection of the complete genome. Thus, the presence and assistance of the PRD1 membrane may constitute a biological advantage that facilitates the infection process. This benefit may be common to many viruses that possess an internal vesicle enclosing densely packaged linear dsDNA.

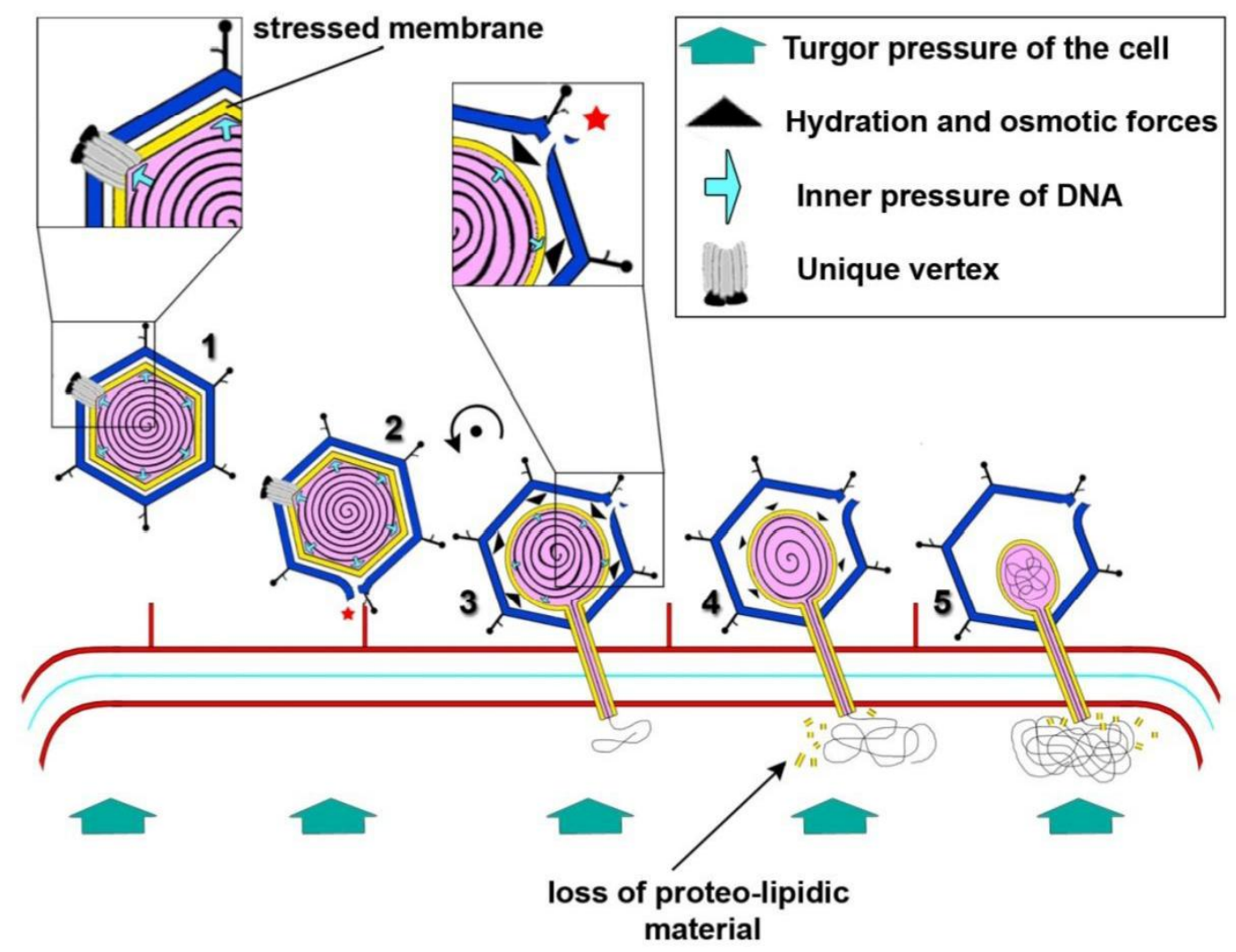

Fig. 6. A proposed model for the membrane-assisted DNA ejection of PRD1.

(1) At the pre-infection stage, the pressure of the packaged DNA is high, 52 atm (inset, cyan arrows), and it maintains a membrane lateral stress of 6.4 MPa.

(2) After adsorption to a receptor, the receptor complex at that vertex is loosened (red star). 
(3) The phage repositions (black curved arrow and dot) with the unique vertex towards the cell surface. The vesicle (yellow) remodels and self-assembles to form a proteo-lipidic tube which then penetrates the outer membrane, cell wall, and cell membrane. As DNA release begins, other forces, including hydration and osmotic pressure (inset, black triangles) contribute to the ejection. The lateral stress of the membrane decreases, thereby generating an additional force driving genome release.

(4-5) Decrease of the membrane radius accompanies loss of proteo-lipidic material from the tip of the tube and the declining internal pressure as the amount of DNA remaining inside decreases. The turgor pressure (green arrows) opposes the ejection force and slows entry of the phage genome. 


\section{Acknowledgments}

We thank David Gil-Carton and Sandra Delgado at the CIC bioGUNE Electron Microscopy Platform Unit for help in virus imaging, and Sari Korhonen and Helin Veskiväli at the ESFRI Instruct Centre for Virus Production (ICVIR), University of Helsinki, for skilled technical assistance in virus production and purification. We also acknowledge the support of employees and the use of experimental resources of Instruct.

The authors thank Academy of Finland (funding grant 272853) and University of Helsinki for the support to EU ESFRI ICVIR used in this study. Isaac Santos-Pérez's internship at the ICVIR was sponsored by Instruct and CIMO Fellowship TM-15-9735. This research received funding by Academy of Finland (Academy Professor funding grants 283072 and 255342 to D.H.B.), by the Spanish Ministerio de Economia y Competitividad (FIS2011-22603 and BioFiVinet FIS2011-16090-E to D.R.; BFU2012-36241 to F.M.G.; BFU201233947/BFU2015-64541-R to N.G.A.A.) and by the Basque Department of Education, Language policy and Culture (to N.G.A.A. and I.S-P). D.R. and N.G.A.A. are members of the Spanish Network of Excellence in Physical Virology - FIS2015-71108-REDT.

\section{Data deposition}

The three tomograms represented in Fig. 2 have been deposited in the EMDataBank with the following codes: XXXX, YYYY, ZZZZ.

\section{Competing interests}

The authors declare that they have no competing interests. 


\section{Author Contributions}

N.G.A.A. conceived the study. N.G.A.A. and D.R. design the experiments. H.M.O. and D.H.B. produced the biologicals. F.M.G. provided insights into membrane biophysics. D.R. performed the modeling studies and calculations. I.S-P. performed the analysis of PRD1 tomograms and cryo-EM reconstructions. All authors contributed to the analysis of the data and final interpretation. I.S-P., D.R. and N.G.A.A. wrote the manuscript with contributions from other authors. 


\section{REFERENCES}

[1] J. Kindt, S. Tzlil, A. Ben-Shaul, W.M. Gelbart, DNA packaging and ejection forces in bacteriophage, Proc Natl Acad Sci U S A, 98 (2001) 13671-13674.

[2] S. Ghosal, Capstan friction model for DNA ejection from bacteriophages, Phys Rev Lett, 109 (2012) 248105.

[3] P.K. Purohit, M.M. Inamdar, P.D. Grayson, T.M. Squires, J. Kondev, R. Phillips, Forces during bacteriophage DNA packaging and ejection, Biophys J, 88 (2005) 851-866.

[4] D. Panja, I.J. Molineux, Dynamics of bacteriophage genome ejection in vitro and in vivo, Physical biology, 7 (2010) 045006.

[5] A. Evilevitch, L. Lavelle, C.M. Knobler, E. Raspaud, W.M. Gelbart, Osmotic pressure inhibition of DNA ejection from phage, Proc Natl Acad Sci U S A, 100 (2003) 9292-9295.

[6] W.M. Gelbart, C.M. Knobler, Virology. Pressurized viruses, Science, 323 (2009) 1682-1683.

[7] S. Marion, A. Siber, Ejecting phage DNA against cellular turgor pressure, Biophys J, 107 (2014) 1924-1929.

[8] M. Jeembaeva, B. Jonsson, M. Castelnovo, A. Evilevitch, DNA heats up: energetics of genome ejection from phage revealed by isothermal titration calorimetry, J Mol Biol, 395 (2010) 1079-1087.

[9] M.M. Inamdar, W.M. Gelbart, R. Phillips, Dynamics of DNA ejection from bacteriophage, Biophys J, 91 (2006) 411-420.

[10] R. Zandi, D. Reguera, J. Rudnick, W.M. Gelbart, What drives the translocation of stiff chains?, Proc Natl Acad Sci U S A, 100 (2003) 8649-8653.

[11] I.J. Molineux, No syringes please, ejection of phage T7 DNA from the virion is enzyme driven, Mol Microbiol, 40 (2001) 1-8.

[12] S.B. Zimmerman, L.D. Murphy, Macromolecular crowding and the mandatory condensation of DNA in bacteria, FEBS Lett, 390 (1996) 245-248.

[13] P.G. Leiman, P.R. Chipman, V.A. Kostyuchenko, V.V. Mesyanzhinov, M.G. Rossmann, Three-dimensional rearrangement of proteins in the tail of bacteriophage T4 on infection of its host, Cell, 118 (2004) 419-429.

[14] X. Fu, M.H. Walter, A. Paredes, M.C. Morais, J. Liu, The mechanism of DNA ejection in the Bacillus anthracis spore-binding phage $8 \mathrm{a}$ revealed by cryo-electron tomography, Virology, 421 (2011) 141-148.

[15] S. Spinelli, D. Veesler, C. Bebeacua, C. Cambillau, Structures and host-adhesion mechanisms of lactococcal siphophages, Frontiers in microbiology, 5 (2014) 3.

[16] I.J. Molineux, D. Panja, Popping the cork: mechanisms of phage genome ejection, Nat Rev Microbiol, 11 (2013) 194-204.

[17] B. Peralta, D. Gil-Carton, D. Castano-Diez, A. Bertin, C. Boulogne, H.M. Oksanen, D.H. Bamford, N.G. Abrescia, Mechanism of membranous tunnelling nanotube formation in viral genome delivery, PLoS Biol, 11 (2013) e1001667.

[18] N.G. Abrescia, J.J. Cockburn, J.M. Grimes, G.C. Sutton, J.M. Diprose, S.J. Butcher, S.D. Fuller, C. San Martin, R.M. Burnett, D.I. Stuart, D.H. Bamford, J.K. Bamford, Insights into assembly from structural analysis of bacteriophage PRD1, Nature, 432 (2004) 68-74.

[19] J.J. Cockburn, N.G. Abrescia, J.M. Grimes, G.C. Sutton, J.M. Diprose, J.M. Benevides, G.J. Thomas, Jr., J.K. Bamford, D.H. Bamford, D.I. Stuart, Membrane structure and interactions with protein and DNA in bacteriophage PRD1, Nature, 432 (2004) 122-125.

[20] A.M. Grahn, R. Daugelavicius, D.H. Bamford, Sequential model of phage PRD1 DNA delivery: active involvement of the viral membrane, Mol Microbiol, 46 (2002) 1199-1209. 
[21] C. Hong, H.M. Oksanen, X. Liu, J. Jakana, D.H. Bamford, W. Chiu, A structural model of the genome packaging process in a membrane-containing double stranded DNA virus, PLoS Biol, 12 (2014) e1002024.

[22] J.K. Bamford, D.H. Bamford, Large-scale purification of membrane-containing bacteriophage PRD1 and its subviral particles, Virology, 181 (1991) 348-352.

[23] D.N. Mastronarde, Automated electron microscope tomography using robust prediction of specimen movements, J Struct Biol, 152 (2005) 36-51.

[24] J.R. Kremer, D.N. Mastronarde, J.R. Mclntosh, Computer visualization of threedimensional image data using IMOD, J Struct Biol, 116 (1996) 71-76.

[25] J.J.L. Fernandez, S., An improved algorithm for anisotropic nonlinear diffusion for denoising cryotomograms, Journal of Structural Biology, 144:152-161, 2003. (2003).

[26] E.F. Pettersen, T.D. Goddard, C.C. Huang, G.S. Couch, D.M. Greenblatt, E.C. Meng, T.E. Ferrin, UCSF Chimera--a visualization system for exploratory research and analysis, J Comput Chem, 25 (2004) 1605-1612.

[27] C.A. Schneider, W.S. Rasband, K.W. Eliceiri, NIH Image to ImageJ: 25 years of image analysis, Nat Methods, 9 (2012) 671-675.

[28] J.M. de la Rosa-Trevin, J. Oton, R. Marabini, A. Zaldivar, J. Vargas, J.M. Carazo, C.O. Sorzano, Xmipp 3.0: an improved software suite for image processing in electron microscopy, J Struct Biol, 184 (2013) 321-328.

[29] P.K. Purohit, J. Kondev, R. Phillips, Mechanics of DNA packaging in viruses, Proc Natl Acad Sci U S A, 100 (2003) 3173-3178.

[30] S. Tzlil, J.T. Kindt, W.M. Gelbart, A. Ben-Shaul, Forces and pressures in DNA packaging and release from viral capsids, Biophys J, 84 (2003) 1616-1627.

[31] S.C. Riemer, V.A. Bloomfield, Packaging of DNA in bacteriophage heads: some considerations on energetics, Biopolymers, 17 (1978) 785-794.

[32] T. Odijk, Hexagonally packed DNA within bacteriophage T7 stabilized by curvature stress, Biophys J, 75 (1998) 1223-1227.

[33] A.S. Petrov, S.C. Harvey, Packaging double-helical DNA into viral capsids: structures, forces, and energetics, Biophys J, 95 (2008) 497-502.

[34] D.C. Rau, V.A. Parsegian, Direct measurement of the intermolecular forces between counterion-condensed DNA double helices. Evidence for long range attractive hydration forces, Biophys J, 61 (1992) 246-259.

[35] D.S. Cayley, H.J. Guttman, M.T. Record, Jr., Biophysical characterization of changes in amounts and activity of Escherichia coli cell and compartment water and turgor pressure in response to osmotic stress, Biophys J, 78 (2000) 1748-1764.

[36] W. Helfrich, Elastic properties of lipid bilayers: theory and possible experiments, Zeitschrift fur Naturforschung. Section C: Biosciences, 28 (1973) 693-703.

[37] L.D.L. Landau, E.M. , Theory of Elasticity Pergamon Press, Volume 7 of A Course of Theoretical Physics (1970).

[38] I.L. Ivanovska, P.J. de Pablo, B. Ibarra, G. Sgalari, F.C. MacKintosh, J.L. Carrascosa, C.F. Schmidt, G.J. Wuite, Bacteriophage capsids: tough nanoshells with complex elastic properties, Proc Natl Acad Sci U S A, 101 (2004) 7600-7605.

[39] W. Helfrich, Out-of-plane fluctuations of lipid bilayers, Zeitschrift fur Naturforschung. Section C: Biosciences, 30 (1975) 841-842.

[40] C. San Martin, J.T. Huiskonen, J.K. Bamford, S.J. Butcher, S.D. Fuller, D.H. Bamford, R.M. Burnett, Minor proteins, mobile arms and membrane-capsid interactions in the bacteriophage PRD1 capsid, Nat Struct Biol, 9 (2002) 756-763. 
[41] S.J. Butcher, D.H. Bamford, S.D. Fuller, DNA packaging orders the membrane of bacteriophage PRD1, Embo J, 14 (1995) 6078-6086.

[42] L. Picas, F. Rico, S. Scheuring, Direct measurement of the mechanical properties of lipid phases in supported bilayers, Biophys J, 102 (2012) L01-03.

[43] M. Arnoldi, M. Fritz, E. Bauerlein, M. Radmacher, E. Sackmann, A. Boulbitch, Bacterial turgor pressure can be measured by atomic force microscopy, Physical review. E, Statistical physics, plasmas, fluids, and related interdisciplinary topics, 62 (2000) 1034-1044.

[44] Y. Deng, M. Sun, J.W. Shaevitz, Direct measurement of cell wall stress stiffening and turgor pressure in live bacterial cells, Phys Rev Lett, 107 (2011) 158101.

[45] D. Gil-Carton, S.T. Jaakkola, D. Charro, B. Peralta, D. Castano-Diez, H.M. Oksanen, D.H. Bamford, N.G. Abrescia, Insight into the Assembly of Viruses with Vertical Single beta-barrel Major Capsid Proteins, Structure, 23 (2015) 1866-1877.

[46] C.Y. Fu, J.E. Johnson, Structure and cell biology of archaeal virus STIV, Curr Opin Virol, 2 (2012) 122-127.

[47] N.G. Abrescia, J.M. Grimes, H.M. Kivela, R. Assenberg, G.C. Sutton, S.J. Butcher, J.K. Bamford, D.H. Bamford, D.I. Stuart, Insights into virus evolution and membrane biogenesis from the structure of the marine lipid-containing bacteriophage PM2, Mol Cell, 31 (2008) 749-761.

[48] M. Castelnovo, A. Evilevitch, DNA ejection from bacteriophage: towards a general behavior for osmotic-suppression experiments, The European physical journal. E, Soft matter, 24 (2007) 9-18.

[49] R. Hauser, S. Blasche, T. Dokland, E. Haggard-Ljungquist, A. von Brunn, M. Salas, S. Casjens, I. Molineux, P. Uetz, Bacteriophage protein-protein interactions, Adv Virus Res, 83 (2012) 219-298.

[50] H.M. Kivela, R. Daugelavicius, R.H. Hankkio, J.K. Bamford, D.H. Bamford, Penetration of membrane-containing double-stranded-DNA bacteriophage PM2 into Pseudoalteromonas hosts, J Bacteriol, 186 (2004) 5342-5354.

[51] N. Zauberman, Y. Mutsafi, D.B. Halevy, E. Shimoni, E. Klein, C. Xiao, S. Sun, A. Minsky, Distinct DNA exit and packaging portals in the virus Acanthamoeba polyphaga mimivirus, PLoS Biol, 6 (2008) e114.

[52] J.L. Van Etten, L.C. Lane, D.D. Dunigan, DNA viruses: the really big ones (giruses), Annu Rev Microbiol, 64 (2010) 83-99. 